\title{
Radiation therapy in management of sporadic and neurofibromatosis type 1-associated malignant peripheral nerve sheath tumors
}

\author{
Jenna Kahn ${ }^{1,2}$, Andy Gillespie ${ }^{3}$, Maria Tsokos $^{4}$, John Ondos ${ }^{1}$, Eva Dombi $^{3}$, Kevin Camphausen ${ }^{1}$, \\ Brigitte C. Widemann ${ }^{3}$ and Aradhana Kaushal ${ }^{1 *}$
}

${ }^{1}$ Radiation Oncology Branch, National Cancer Institute, National Institutes of Health, Bethesda, MD, USA

${ }^{2}$ Warren Alpert School of Medicine, Brown University, Providence, RI, USA

${ }^{3}$ Pediatric Oncology Branch, National Cancer Institute, National Institutes of Health, Bethesda, MD, USA

${ }^{4}$ Department of Pathology, National Institutes of Health, Bethesda, MD, USA

\section{Edited by:}

Anatoly Dritschilo, Georgetown

University School of Medicine, USA

Reviewed by:

John Austin Vargo, University of

Pittsburgh Cancer Institute, USA

Yidong Yang, University of Miami

Miller School of Medicine, USA

*Correspondence:

Aradhana Kaushal, Radiation

Oncology Branch, National Cancer

Institute, National Institutes of Health,

Building 10-CRC, Room B2-3500, 10

Center Drive, Bethesda, MD 20892,

USA

e-mail:kaushala@mail.nih.gov
Introduction: Malignant peripheral nerve sheath tumors (MPNSTs) are highly aggressive soft tissue sarcomas in which complete surgical resection is the mainstay of therapy. However, the recurrence rate is high and few options remain for refractory or metastatic MPNST. This study examines the outcomes of adjuvant radiation therapy in MPNST in patients with and without neurofibromatosis type 1 (NF1) and reviews the literature on use of radiation for MPNST.

Methods: A retrospective review of 33 MPNST patients between 1990 and 2012 evaluated at the $\mathrm{NIH}$. All diagnoses were pathologically confirmed at the $\mathrm{NCl}$. Clinical presentation, treatment, and survival were analyzed.

Results: Thirty-three patients were included 18 NF1-associated, 15 sporadic tumors. Tumor location included extremity (58\%), trunk (36\%), and head/neck (6\%). Histologic grade showed 25 high-grade tumors compared to 7 low-grade tumors. Twenty patients were treated with radiation therapy (median total dose of $58.5 \mathrm{~Gy}$ with $1.8 \mathrm{~Gy} /$ fraction). A median survival of all patients was 46.5 months and $43.7 \%$ overall 5 -year survival. Prognostic factors include extent of resection, tumor location, and histology grade. Radiation was not found to be a prognostic factor for overall survival.

Conclusion: This study is consistent with previous studies regarding the role of radiation in the management of MPNST. Prospective evaluation of adjuvant radiation will allow to more fully define the role of radiation in MPNST.

Keywords: malignant peripheral nerve sheath tumor, malignant schwannoma, neurofibrosarcoma, neurogenic sarcoma, radiation therapy

\section{INTRODUCTION}

Malignant peripheral nerve sheath tumors (MPNSTs), also known as malignant schwannomas, neurofibrosarcomas, and neurogenic sarcomas, are rare, highly aggressive malignancies that arise from major or minor peripheral nerve branches or sheaths. They account for about $10 \%$ of soft tissue sarcomas $(1,2)$. More than half of these malignancies arise in individuals with neurofibromatosis type 1 (NF1). The majority of NF1-associated MPNSTs arise from preexisting plexiform neurofibromas. The lifetime incidence of MPNSTs in NF1 patients is $8-13 \%$ whereas the general population has an incidence of $0.01 \%(1,3-8)$. Several studies have shown that patients with NF1-associated tumors have a worse disease specific survival compared with sporadic tumors $(1,3,9-12)$.

Complete surgical resection of MPNSTs is required for cure. Even with aggressive surgery and negative surgical margins, local and distant recurrence occurs frequently. Radiation and chemotherapy are additional modalities that are used to treat these tumors. However, poor response to standard sarcoma chemotherapy has been described for NF1-associated MPNST $(1,13)$. Despite current multimodality therapy, the 5-year survival ranges from 35 to $50 \%(1,3,6,7)$. The role of radiation therapy in MPNST is still in evolution. Many studies have recommended the use of radiation therapy in an adjuvant setting $(1,3,4,10,11,14-19)$. Yet, only one study, to date, has shown a statistically significant increased local control with radiation therapy (10). Thus, the primary goal of this study was to examine the role and utility of adjuvant radiation therapy in patients evaluated at the NCI for sporadic and NF1associated MPNST. A secondary goal was to review the literature of the use of radiation therapy in MPNST.

\section{MATERIALS AND METHODS}

Medical records of patients evaluated at the National Cancer Institute between January 1990 and June 2012 for potential diagnosis of MPNST were retrieved. For inclusion in this study, MPNST histological diagnosis was confirmed by a NCI pathologist. This was 
approved by the office of human subjects research protection as a retrospective analysis.

Malignant peripheral nerve sheath tumors were categorized as NF1-associated in patients with a clinical diagnosis of NF1 using consensus criteria or sporadic in the absence of a diagnosis of NF1 (20). Clinicopathologic data collected included age at initial diagnosis, gender, tumor location, tumor size, presence of metastases, extent of resection, histology grade, immunohistochemical staining for S100, and vimentin, neoadjuvant, and/or adjuvant chemotherapy, and/or radiation. Histology grade was defined as low or high grade. Low-grade referred to as grade I and high as grade II and III (21). Tumor location were defined as extremity (upper and lower), trunk (chest wall, proximal groin, thorax, abdomen, and retroperitoneum), or head and neck. Extent of resection were defined as R0 (negative margins), R1 (microscopically positive), and R2 (macroscopically positive) as defined in the pathology and surgical reports.

Radiation was defined as a treatment with curative intent. Radiation dose, fractions, field size, treatment breaks, and toxicities, resulting from radiation were also included. Acute toxicities were judged by radiation therapy oncology group (RTOG) criteria.

Overall survival (OS), the time from diagnosis to death, and the influence of clinicopathologic features on OS and local control was analyzed using the Kaplan-Meier method and using the log-rank (Mantel-Cox) test in the univariate setting. Statistical significance was defined as $p<0.05$.

A comprehensive review of the literature for treatment of MPNST was conducted. The systematic literature search included Cochrane Collaboration Library electronic database, PubMed, RTOG.org, and ClinicalTrials.gov, using the following terms and keywords: MPNST, NF1, neurofibrosarcoma, malignant schwannoma, radiation therapy, and a combination of these terms. Studies were limited to those reported in the English language and human subjects. Original studies were reviewed independently.

\section{RESULTS}

\section{PATIENT AND TUMOR CHARACTERISTICS}

Of the 56 patients evaluated for MPNST, 33 fulfilled criteria for this study (Table 1). Most patients were excluded because there was not enough data or follow up. NF1-associated MPNSTs $(n=18)$ were more frequent than sporadic MPNSTs $(n=15)$. The median overall age at diagnosis was 25 years (range $9-76$ years). There was a difference in median age between NF1 patients and sporadic, 15 and 41, respectively. Females constituted 33\% $(n=11)$ of the study. There were more males in the NF1 study group as compared to the sporadic patients, 78 and 53\%, respectively.

Most tumors presented as local disease but one patient in each group presented with metastatic disease. NF1-associated tumors were larger at the time of diagnosis, $438.4 \mathrm{~cm}^{3}$ (range: $64-4377 \mathrm{~cm}^{3}$ ), whereas the sporadic tumor volume was $240.6 \mathrm{~cm}^{3}$ (range: $10-1000 \mathrm{~cm}^{3}$ ). MPNSTs were most commonly located in the extremity $(n=19,58 \%)$, followed by trunk $(n=12,36 \%)$, and least commonly head and neck region $(n=2,6 \%)$. Both NF1-associated and sporadic tumors were most common in the extremity, 61 and 53\%, respectively.

Histologic grade of these tumors reported that high-grade disease was more prevalent $(n=25)$. The presence of necrosis was
Table 1 | Patient characteristics.

\begin{tabular}{|c|c|c|}
\hline Characteristics & NF1 $(n=18)$ & Sporadic $(n=15)$ \\
\hline Median age (years) & 15 & 41 \\
\hline \multicolumn{3}{|l|}{ Gender } \\
\hline Female & $4(22 \%)$ & $7(47 \%)$ \\
\hline Male & $14(78 \%)$ & $8(53 \%)$ \\
\hline \multicolumn{3}{|l|}{ Presentation } \\
\hline Local & 17 (94\%) & $14(93 \%)$ \\
\hline Metastatic & $1(6 \%)$ & $1(7 \%)$ \\
\hline Tumor size $\left(\mathrm{cm}^{3}\right)$ & 931.4 & 275.6 \\
\hline \multicolumn{3}{|l|}{ Location of tumor } \\
\hline Head/neck & - & $2(13 \%)$ \\
\hline Trunk & 7 (39\%) & $5(33 \%)$ \\
\hline Extremity & $11(61 \%)$ & $8(53 \%)$ \\
\hline \multicolumn{3}{|l|}{ Histology } \\
\hline Low grade & $4(22 \%)$ & $3(20 \%)$ \\
\hline High grade & $14(83 \%)$ & $11(73 \%)$ \\
\hline Presence of necrosis & $9(50 \%)$ & $1(7 \%)$ \\
\hline \multicolumn{3}{|l|}{$\mathrm{S} 100$} \\
\hline Positive & $11(61 \%)$ & $10(67 \%)$ \\
\hline Negative & $1(6 \%)$ & $2(13 \%)$ \\
\hline \multicolumn{3}{|l|}{ Vimentin } \\
\hline Positive & $3(17 \%)$ & $8(53 \%)$ \\
\hline Negative & $3(17 \%)$ & $2(13 \%)$ \\
\hline \multicolumn{3}{|l|}{ Extent of resection } \\
\hline Ro & 7 (39\%) & $6(40 \%)$ \\
\hline $\mathrm{R} 1$ & $4(22 \%)$ & $4(27 \%)$ \\
\hline $\mathrm{R} 2$ & 7 (39\%) & $5(33 \%)$ \\
\hline Neoadjuvant chemotherapy & $2(11 \%)$ & $7(47 \%)$ \\
\hline Radiation & $10(56 \%)$ & $10(67 \%)$ \\
\hline \multicolumn{3}{|l|}{ Treatment regimens } \\
\hline Surgery alone & $4(22 \%)$ & $1(6 \%)$ \\
\hline Chemotherapy + surgery & $4(22 \%)$ & $4(27 \%)$ \\
\hline Radiation + surgery & - & $3(20 \%)$ \\
\hline Chemotherapy + surgery + radiation & $10(56 \%)$ & $7(47 \%)$ \\
\hline
\end{tabular}

seen in nine patients with NF1 (50\%) and one patients with sporadic tumors (7\%). S100 positive staining was seen in $61 \%$ of NF1 patients while sporadic tumors showed $67 \%$ positive staining. Vimentin staining was positive in three patients with NF-associated and eight patients with sporadic tumors.

The extent of resection was similar in both groups. Negative surgical margins were achieved in 13 patients. R1 resection was achieved in 8 patients and R2 resection in 12. A total of 9 patients were treated with neoadjuvant chemotherapy and 20 patients $(61 \%)$ treated with radiation therapy. The most commonly used chemotherapy agents for these patients included vincristine, adriamycin, cyclophosphamide, etoposide, docetaxel, ifosfamide, cisplatin, and gemcitabine. Treatment regimens consisted of surgery $(n=5)$, chemotherapy plus surgery $(n=8)$, radiation plus surgery $(n=3)$, and chemotherapy, radiation, and surgery $(n=17)$. 
Twenty patients were treated with radiation therapy (Table 2). Modalities of radiation included external beam $(n=15)$, brachytherapy $(n=2)$, proton therapy $(n=1)$, and external beam plus brachytherapy $(n=2)$. NF1-associated tumors $(n=10)$ received a median total dose of $59.4 \mathrm{~Gy}$ with a median of $1.8 \mathrm{~Gy} /$ fractions. Sporadic tumors in the adjuvant setting $(n=10)$ received a median total dose of $58.5 \mathrm{~Gy}$ with a median of $1.8 \mathrm{~Gy} /$ fractions. Local control rates for NF-1 patients treated with radiation were $51 \%$ at 5 years. Local control rates for patients treated with radiation were $53 \%$ as compared to $45 \%$ in those not treated with radiation at 5 years, which was not statistically significant. Patients were treated with a mix of IMRT and 3D conformal using $6 \mathrm{MV}$. The field size of the tumor that was treated was substantially larger in NF1-associated tumors as compared to sporadic tumors, $597.9 \mathrm{cc}^{3}$ (range: $31.5-2325.1 \mathrm{cc}^{3}$ ) and $290.9 \mathrm{cc}^{3}$ (range: $18-486.5 \mathrm{cc}^{3}$ ), respectively. One patient from each NF1-associated and sporadic tumors required a treatment break during radiation. RTOG acute grade I toxicities were reported in a total of eight patients, three patients in the NF1 and five patients in the sporadic groups. Toxicities included fatigue, nausea, mild dysphagia, mild erythema, mild odynophagia, and desquamation. There were no toxicities exceeding grade II. Secondary cancers occurred in three patients including pilocytic astrocytoma, brain cancer (unspecified), and liver. Two of these patients had NF1 and the liver cancer was in the field of radiation treatment.

\section{SURVIVAL ANALYSIS}

The median survival of all patients was 33 months and overall 5year survival was $43.7 \%$ (Table 3; Figure 1). In the NF1-associated tumors, the OS was 22.1 months as compared to sporadic tumors with a median of 64.3 months $(p=0.13)$ (Figure 2A). NF1associated tumors treated with radiation had a median survival of 33.1 months as compared to 17.4 months with those patients that did not receive radiation treatment. Distant metastases accounted for five patients (28\%) in NF1-associated MPNST as compared to seven patients in the sporadic MPNST (47\%). Pulmonary metastasis was seen in three patients $(17 \%)$ in the NF1-associated and seven patients $(47 \%)$ in the sporadic MPNST. Poor prognostic factors that were statistically significant included incomplete resection $(p=0.007)$ (Figure $2 \mathbf{B})$, histologic grade $(p=0.04)$, and truncal tumor location $(p=0.01)$ (Figure 2C). Radiation therapy was not found to be a prognostic factor for OS $(p=0.97)$ (Figure 2D).

\section{LITERATURE REVIEW}

The literature review identified 19 mostly retrospective studies published since 1973 with patients treated starting in 1920 until 2011. The studies included 20-205 patients with $17-100 \%$ with NF1. Tumor size as a prognostic factor for survival was significant in 12 studies where larger tumors had a worse prognosis. NF1 was regarded as a negative prognostic factor in seven studies. Age at initial diagnosis, where younger age was a worse prognosis for survival, was significant for four studies and tumor location for nine studies. Extent of resection was a prognostic factor in 14 studies, where incomplete resection had a worse survival prognosis. Radiation was a statistically significant local control prognostic factor in one study (10), yet, there was a trend and recommendation for
Table 2 | Radiation characteristics

\begin{tabular}{lcc}
\hline Characteristics & NF1 $(\boldsymbol{n}=\mathbf{1 0})$ & Sporadic $(\boldsymbol{n}=\mathbf{1 0})$ \\
\hline Type of radiation & & \\
External beam & $7(70 \%)$ & $8(80 \%)$ \\
Brachytherapy & $1(10 \%)$ & $1(10 \%)$ \\
Proton therapy & $1(10 \%)$ & 0 \\
External beam and brachytherapy & $1(10 \%)$ & $1(10 \%)$ \\
Adjuvant radiation & & \\
Fractions (median) & 31 & 28 \\
Dose/fraction (median) & $1.8 \mathrm{~Gy}$ & $1.8 \mathrm{~Gy}$ \\
$\quad$ Total dose (median) & $59.4 \mathrm{~Gy}$ & $58.5 \mathrm{~Gy}$ \\
Treatment volume (cc $\left.{ }^{3}\right)$ (mean) & 438.4 & 240.6 \\
Treatment breaks & 1 & 1 \\
RTOG acute grade toxicities & 3 & 5 \\
\hline
\end{tabular}

Table 3 | Survival of NF1 and sporadic MPNST.

\begin{tabular}{lcc}
\hline Characteristics & NF1 $(\boldsymbol{n}=\mathbf{1 8})$ & Sporadic $(\boldsymbol{n}=\mathbf{1 5})$ \\
\hline Overall survival (median) & 22.1 months & 64.3 months \\
Radiation & 33.1 months & 51.6 months \\
No radiation & 17.4 months & 69.4 months \\
Local failure & $7(39 \%)$ & $5(33 \%)$ \\
Distant failure & $5(28 \%)$ & $7(47 \%)$ \\
Pulmonary metastasis & $3(17 \%)$ & $7(47 \%)$
\end{tabular}

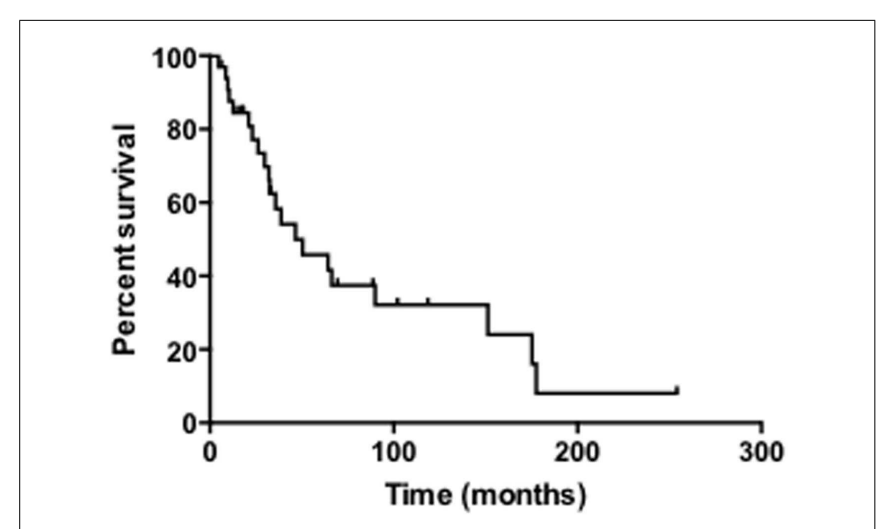

FIGURE 1 | Overall survival of patients with MPNST.

radiation in seven reports. Overall of the 19 studies, $18-83 \%$ of patients received radiation therapy treatment. Doses ranged from $12.5-90$ Gy in these studies. As seen in the table, OS ranged from 16 to $65.7 \%$ (Table 4 ).

\section{DISCUSSION}

Malignant peripheral nerve sheath tumors are uncommon soft tissue sarcomas. The prognosis for unresectable MPNST is poor. There is a scarcity of data addressing radiation therapy as a local 

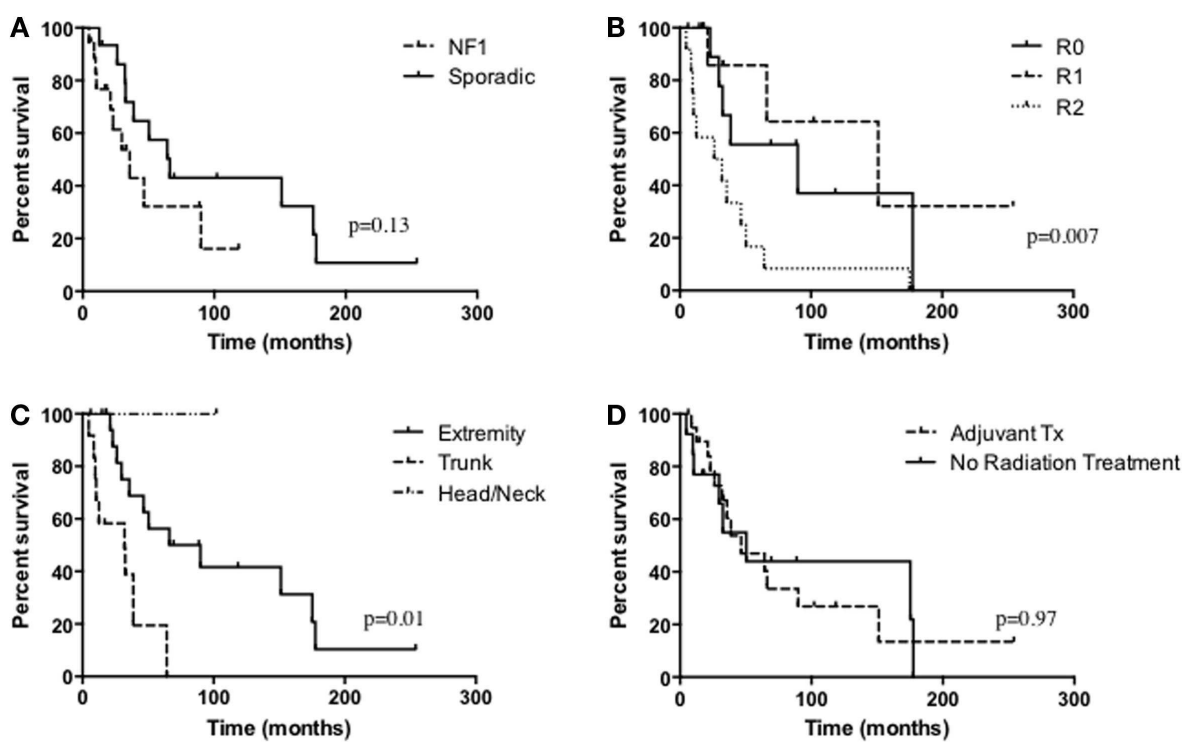

FIGURE 2 | Overall survival of (A) NF1-associated MPNST compared with sporadic MPNST, $p=0.13$; $(B)$ the extent of resection, $p=0.007$; (C) tumor location, $p=0.01$; $(D)$ radiation treatment versus no radiation treatment, $p=0.97$.

therapy. Our study provides a detailed analysis of the treatment of patients with sporadic or NF1-associated MPNSTs as well as a review of the literature.

The association between MPNST and NF1 has been documented extensively. Studies have shown that individuals with NF1 have a higher than expected frequency of MPNST $(3,9,10,14,27)$. This may be because patients with NF1 have a somatic mutation in the NF1 tumor suppressor gene, which results in the development of benign nerve sheath tumors called plexiform neurofibromas, which are at risk for malignant degeneration (28). In many studies, the mean age of diagnosis of MPNST in these NF1 patients is between 25 and 28 years old (2-4). As seen in the literature age at diagnosis has also been a prognostic factor for some studies including Wanebo et al. who found that there was reduced survival in patients younger than 30 years of age reflecting on the aggressiveness in the younger population (4). Our study similarly showed a median diagnosis of 15 years old. In sporadic tumors, the mean age of diagnosis for patients has ranged from 39 to 60 in studies (2-4). Our study likewise shows a median of 41 years old for sporadic tumors.

Some studies have shown that NF1 was a poor prognostic factor for survival statistically while others have shown a trend toward sporadic tumors being a good prognostic indicator (1, 3, 9-12, $15,18,22)$. The 5 -year survival rates of those with NF1-associated tumors ranged from 16 to $60 \%$, whereas in sporadic tumors the rates ranged from 47 to $75 \%(1,3,9-11,16,22)$. At diagnosis, NF1associated MPNST tend to be large, invasive, and unresectable (1). NF1-associated MPNSTs are commonly more frequent in the trunk as compared to sporadic MPNST $(6,11)$, which affects outcome because central lesions may be less amenable to surgery than are extremity MPNSTs $(3,22)$. NF1-associated MPNSTs have also been thought to have a greater tendency to metastasize than sporadic MPNSTs. Ducatman et al. showed that of 62 patients with NF1-associated MPNSTs, 39\% developed metastatic disease whereas only $16 \%$ of 58 sporadic MPNSTs developed metastases (3). The diagnosis of MPNST in patients with NF1 is difficult to establish clinically without biopsy because they are often mistaken for neurofibromas and therefore present at a later stage $(3,15$, 29 ). NF1-associated tumors had a 5 -year OS of $32.2 \%$ as compared with $53.8 \%$ in sporadic tumors. Although our data does not show statistical significance ( $p=0.13$ ) for poor prognosis of NF1 patients our data does show a trend that sporadic patients have a better prognosis. Our data correlate with these studies and suggest that the need for expeditious evaluation in patients with NF1 with rapidly growing lesions.

Tumor location has been hypothesized as a prognostic factor because of the ability of complete surgical resection to be more easily achieved in extremities versus tumors in the abdomen or chest $(3,22)$. Primary tumors of the extremities are also thought to be diagnosed earlier because the more visible location $(10,22)$. Tumor location was found to be a prognostic factor in nine studies with extremities having the better prognosis. The location of the tumor was found to be a prognostic factor for survival in our study $(p=0.01)$. Tumors located in the truncal region fared worse than those with lesions in the extremities, which is consistent with the literature.

Malignant peripheral nerve sheath tumors are locally aggressive and failure to achieve local control remains the major cause of treatment failure. Complete surgical resection is the only curative treatment for sporadic and NF1-associated MPNST (1). The surgical goal is to resect the tumor with wide negative margins. In our study as well as previous studies, incomplete resection and negative margins have been shown to be statistically significant for survival $(1,3,4,6,7,9-11,14,16-18,23,26)$. The results of this study indicate that complete surgical removal of the tumor is the mainstay of treatment and a strong predictor of survival. 


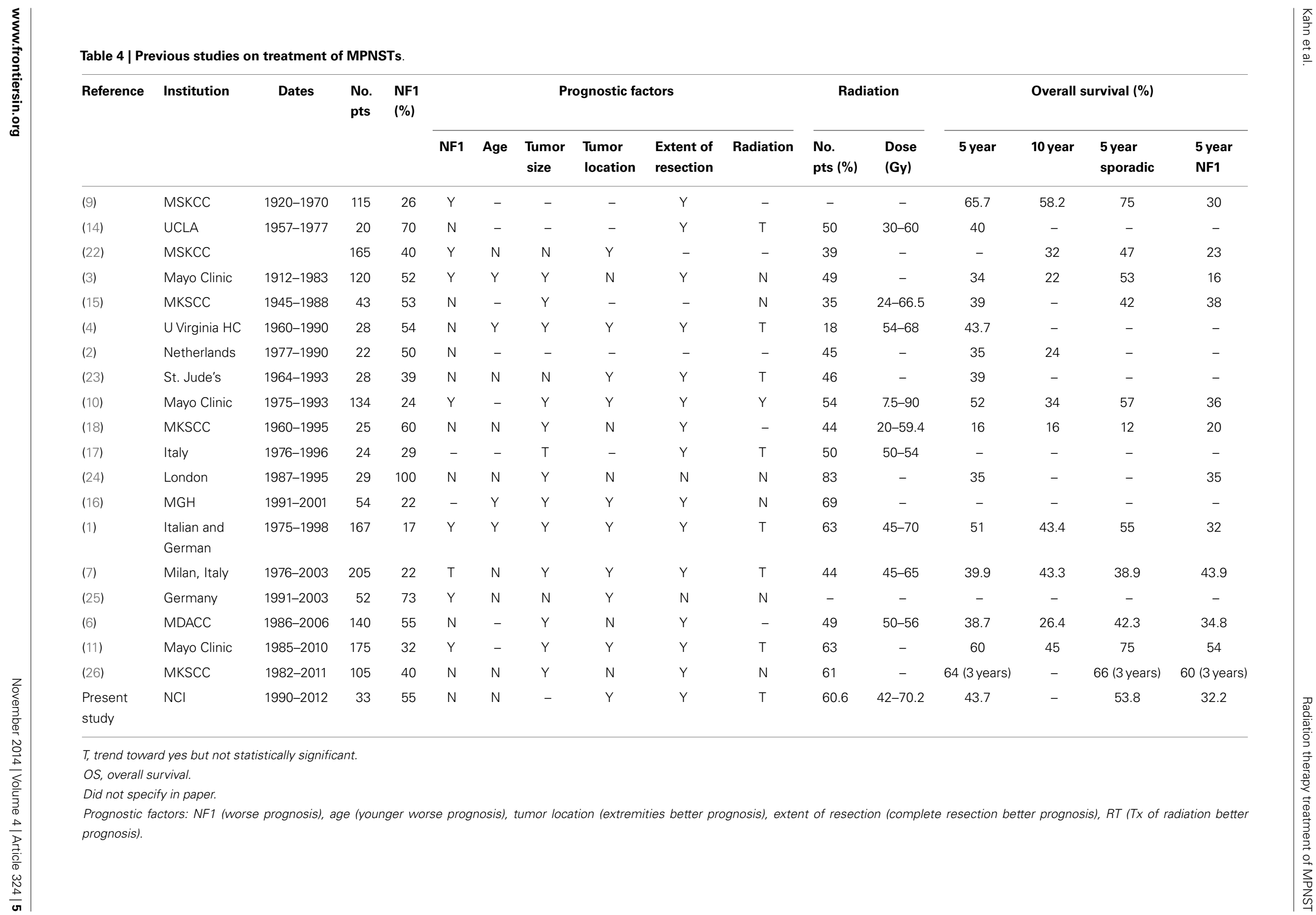


Multidisciplinary evaluation of these patients is crucial in order to provide optimal care. Numerous studies have not found radiation therapy to be a prognostic factor for local control or survival $(1,3,4,6-8,11,12,15,16,18,22-25)$. Yet, many studies have also stated that the use of adjuvant therapy is expanding when patients do not have clear surgical margins $(1,4,7,30)$. Although there have been no prospective randomized trials of radiation therapy in the context of MPNST, there have been studies of the role of adjuvant radiation in adult soft tissue sarcomas $(31,32)$. Yang et al. found that post-operative radiation therapy is highly effective in local control in soft tissue sarcomas of the extremities (31). Wong et al. found that the 5-year local control rates for radiation doses higher than 60 Gy was $73 \%$ as compared with $50 \%$ for lower doses $(p<0.021)$, showing that post-operative radiation therapy has a role in improving local control $(10,17)$. Yet, in an updated study of Stucky et al. with some of the patients from the Wong et al. study as well as two more institutions, the analysis showed radiation therapy not to be a prognostic factor for local control. It was hypothesized that this may be because of an evolution of treatment with an emphasis of achieving negative margins. Even so, Stucky et al. encouraged that radiation therapy be utilized for tumors that have aggressive features including size $\geq 5 \mathrm{~cm}$, high grade, and R1 or R2 margin status (11). Our study similarly showed that the patients treated with adjuvant radiation were not a prognostic factor for local control. This study should also take into account that there is an inherent selection bias given the retrospective nature of the study. Patients were likely selected for radiation therapy based on more extensive tumors, margin status, and unresponsiveness to other therapies, which would bias the results toward not showing a benefit of radiotherapy. As evident by these studies, the role of radiation therapy is still being defined.

In this series, we evaluated the effectiveness of radiation therapy on survival in the setting of MPNST. Radiation therapy treatment was not statistically significant for OS in our study. These data are consistent with the literature where there is no MPNST study that shows a statistically significant difference in OS for those with radiation treatment. In a SEER analysis, high-grade tumors treated with radiation did have improved survival where they looked at 142 patients with MPNST and grouped them with other soft tissue sarcomas (33). Although there have not been studies only looking at MPNSTs, this study would infer that high-grade soft tissue sarcomas, including MPNST, should be treated with radiation therapy. Aggressive therapeutic approaches are important in patients at high risk and every effort should be considered.

In our study, most patients treated with radiation received external beam therapy while few patients received protons and brachytherapy. In the adjuvant setting, the total radiation dose was $\geq 56 \mathrm{~Gy}$. This is compared with previous studies, which have found that a cumulative dose of $\geq 60$ Gy was required to provide local disease control $(10,32)$. Although very few patients were treated with brachytherapy this modality was given as a boost to external beam radiation. Previous studies have evaluates the use of brachytherapy for soft tissue sarcomas and found it to be an effective treatment in combination with external beam radiation in local control (34). One study, in particular, found the use of brachytherapy or intraoperative electron radiation therapy to be a prognostic factor for local control with a 5-year local control of $88 \%$ in those treated with brachytherapy and 51\% in those treated with external beam (10). Adjuvant external beam therapy in another study was found to have better local control then brachytherapy (35). Only one patient in our study received proton therapy and is alive without evidence of disease. Even though there have been no studies on proton beam therapy in MPNSTs this modality of treatment may be a viable option for younger patients or to minimize toxicity.

\section{CONCLUSION}

In conclusion, our analysis shows that radiation therapy can be effective in achieving local and symptomatic control with welltolerated toxicities. Prognostic factors for survival include tumor location, histology, and extent of surgical resection. Our study is limited by its retrospective nature, small sample size, and heterogeneity in the patient population analyzed and their treatment. Prospective studies evaluating the effect of radiation therapy is required. In the adjuvant setting, it will be imperative to assess the dose and modality of treatment. The role of radiosensitizers and other novel techniques should also be explored.

\section{REFERENCES}

1. Carli M, Ferrari A, Mattke A, Zanetti I, Casanova M, Bisogno G, et al. Pediatric malignant peripheral nerve sheath tumor: the Italian and German soft tissue sarcoma cooperative group. J Clin Oncol (2005) 23:8422-30. doi:10.1200/JCO. 2005.01.4886

2. Doorn PF, Molenaar WM, Buter J, Hoekstra HJ. Malignant peripheral nerve sheath tumors in patients with and without neurofibromatosis. Eur J Surg Oncol (1995) 21:78-82. doi:10.1016/S0748-7983(05)80073-3

3. Ducatman BS, Scheithauer BW, Piepgras DG, Reiman HM, Ilstrup DM. Malignant peripheral nerve sheath tumors. A clinicopathologic study of 120 cases. Cancer (1986) 57:2006-21. doi:10.1002/1097-0142(19860515)57: $10<2006:: A I D-C N C R 2820571022>3.0 . C O ; 2-6$

4. Wanebo JE, Malik JM, VandenBerg SR, Wanebo HJ, Driesen N, Persing JA. Malignant peripheral nerve sheath tumors. A clinicopathologic study of 28 cases. Cancer (1993) 71:1247-53. doi:10.1002/1097-0142(19930215)71: $4<1247::$ AID-CNCR2820710413>3.0.CO;2-S

5. Kar M, Deo SV, Shukla NK, Malik A, DattaGupta S, Mohanti BK, et al. Malignant peripheral nerve sheath tumors (MPNST) - clinicopathological study and treatment outcome of twenty-four cases. World J Surg Oncol (2006) 4:55. doi:10.1186/1477-7819-4-55

6. Zou C, Smith KD, Liu J, Lahat G, Myers S, Wang WL, et al. Clinical, pathological, and molecular variables predictive of malignant peripheral nerve sheath tumor outcome. Ann Surg (2009) 249:1014-22. doi:10.1097/SLA.0b013e3181a77e9a

7. Anghileri M, Miceli R, Fiore M, Mariani L, Ferrari A, Mussi C, et al. Malignant peripheral nerve sheath tumors: prognostic factors and survival in a series of patients treated at a single institution. Cancer (2006) 107:1065-74. doi:10.1002/cncr.22098

8. Evans DG, Baser ME, McGaughran J, Sharif S, Howard E, Moran A. Malignant peripheral nerve sheath tumours in neurofibromatosis 1. J Med Genet (2002) 39:311-4. doi:10.1136/jmg.39.5.311

9. Ghosh BC, Ghosh L, Huvos AG, Fortner JG. Malignant schwannoma. A clinicopathologic study. Cancer (1973) 31:184-90. doi:10.1002/1097-0142(197301) 31:1<184::AID-CNCR2820310126>3.0.CO;2-8

10. Wong WW, Hirose T, Scheithauer BW, Schild SE, Gunderson LL. Malignant peripheral nerve sheath tumor: analysis of treatment outcome. Int J Radiat Oncol Biol Phys (1998) 42:351-60. doi:10.1016/S0360-3016(98)00223-5

11. Stucky CC, Johnson KN, Gray RJ, Pockaj BA, Ocal IT, Rose PS, et al. Malignant peripheral nerve sheath tumors (MPNST): the mayo clinic experience. Ann Surg Oncol (2012) 19(3):878-85. doi:10.1245/s10434-011-1978-7

12. Moretti VM, Crawford EA, Staddon AP, Lackman RD, Ogilvie CM. Early outcomes for malignant peripheral nerve sheath tumor treated with chemotherapy. Am J Clin Oncol (2011) 34:417-21. doi:10.1097/COC.0b013e3181e9c08a

13. Ferrari A, Miceli R, Rey A, Oberlin O, Orbach D, Brennan B, et al. Non-metastatic unresected paediatric non-rhabdomyosarcoma soft tissue sarcomas: results of a 
pooled analysis from united states and European groups. Eur J Cancer (2011) 47:724-31. doi:10.1016/j.ejca.2010.11.013

14. Storm FK, Eilber FR, Mirra J, Morton DL. Neurofibrosarcoma. Cancer (1980) 45:126-9. doi:10.1002/1097-0142(19800101)45:1<126::AIDCNCR2820450122>3.0.CO;2-U

15. Hruban RH, Shiu MH, Senie RT, Woodruff JM. Malignant peripheral nerve sheath tumors of the buttock and lower extremity. A study of 43 cases. Cancer (1990) 66:1253-65. doi:10.1002/1097-0142(19900915)66:6<1253::AIDCNCR2820660627>3.0.CO;2-R

16. Baehring JM, Betensky RA, Batchelor TT. Malignant peripheral nerve sheath tumor: the clinical spectrum and outcome of treatment. Neurology (2003) 61:696-8. doi:10.1212/01.WNL.0000078813.05925.2C

17. Casanova M, Ferrari A, Spreafico F, Luksch R, Terenziani M, Cefalo G, et al. Malignant peripheral nerve sheath tumors in children: a single-institution twenty-year experience. J Pediatr Hematol Oncol (1999) 21:509-13. doi:10.1097/ 00043426-199911000-00011

18. Kourea HP, Bilsky MH, Leung DH, Lewis JJ, Woodruff JM. Subdiaphragmatic and intrathoracic paraspinal malignant peripheral nerve sheath tumors: a clinicopathologic study of 25 patients and 26 tumors. Cancer (1998) 82:2191-203. doi:10.1002/(SICI)1097-0142(19980601)82:11<2191: :AID-CNCR14>3.0.CO;2-P

19. Smith KB, Indelicato DJ, Knapik JA, Lagmay JP, Morris C, Kirwan JM, et al. Adjuvant radiotherapy for pediatric and young adult nonrhabdomyosarcoma soft-tissue sarcoma. Int J Radiat Oncol Biol Phys (2011) 81:150-7. doi:10.1016/ j.ijrobp.2010.05.005

20. Ferner RE, Gutmann DH. International consensus statement on malignant peripheral nerve sheath tumors in neurofibromatosis. Cancer Res (2002) 62:1573-7.

21. Rodriguez FJ, Folpe AL, Giannini C, Perry A. Pathology of peripheral nerve sheath tumors: diagnostic overview and update on selected diagnostic problems. Acta Neuropathol (2012) 123:295-319. doi:10.1007/s00401-012-0954-z

22. Sordillo PP, Helson L, Hajdu SI, Magill GB, Kosloff C, Golbey RB, et al. Malignant schwannoma - clinical characteristics, survival, and response to therapy. Cancer (1981) 47:2503-9. doi:10.1002/1097-0142(19810515)47:10<2503: :AID-CNCR2820471033>3.0.CO;2-3

23. de Cou JM, Rao BN, Parham DM, Lobe TE, Bowman L, Pappo AS, et al. Malignant peripheral nerve sheath tumors: the St. Jude children's research hospital experience. Ann Surg Oncol (1995) 2:524-9. doi:10.1007/BF02307086

24. Ramanathan RC, Thomas JM. Malignant peripheral nerve sheath tumours associated with von Recklinghausen's neurofibromatosis. Eur J Surg Oncol (1999) 25:190-3. doi:10.1053/ejso.1998.0625

25. Hagel C, Zils U, Peiper M, Kluwe L, Gotthard S, Friedrich RE, et al. Histopathology and clinical outcome of NF1-associated vs. sporadic malignant peripheral nerve sheath tumors. J Neurooncol (2007) 82:187-92. doi:10.1007/s11060-0069266-2

26. LaFemina J, Qin LX, Moraco NH, Antonescu CR, Fields RC, Crago AM, et al. Oncologic outcomes of sporadic, neurofibromatosis-associated, and radiationinduced malignant peripheral nerve sheath tumors. Ann Surg Oncol (2012) 20(1):66-72. doi:10.1245/s10434-012-2573-2
27. Zöller ME, Rembeck B, Odén A, Samuelsson M, Angervall L. Malignant and benign tumors in patients with neurofibromatosis type 1 in a defined Swedish population. Cancer (1997) 79:2125-31. doi:10.1002/(SICI)10970142(19970601)79:11<2125::AID-CNCR9>3.3.CO;2-B

28. Koshy S. Soft-tissue considerations in mandibular setback. Am J Orthod Dentofacial Orthop (2010) 137:447-8. doi:10.1016/j.ajodo.2010.02.011

29. Widemann BC. Current status of sporadic and neurofibromatosis type 1associated malignant peripheral nerve sheath tumors. Curr Oncol Rep (2009) 11:322-8. doi:10.1007/s11912-009-0045-z

30. Basso-Ricci S. Therapy of malignant schwannomas: usefulness of an integrated radiologic. Surgical therapy. J Neurosurg Sci (1989) 33:253-7.

31. Yang JC, Chang AE, Baker AR, Sindelar WF, Danforth DN, Topalian SL, et al. Randomized prospective study of the benefit of adjuvant radiation therapy in the treatment of soft tissue sarcomas of the extremity. J Clin Oncol (1998) 16:197-203.

32. Mundt AJ, Awan A, Sibley GS, Simon M, Rubin SJ, Samuels B, et al. Conservative surgery and adjuvant radiation therapy in the management of adult soft tissue sarcoma of the extremities: clinical and radiobiological results. Int J Radiat Oncol Biol Phys (1995) 32:977-85. doi:10.1016/0360-3016(95)00111-B

33. Koshy M, Rich SE, Mohiuddin MM. Improved survival with radiation therapy in high-grade soft tissue sarcomas of the extremities: a seer analysis. Int J Radiat Oncol Biol Phys (2010) 77:203-9. doi:10.1016/j.ijrobp.2009.04.051

34. Pisters PW, Harrison LB, Leung DH, Woodruff JM, Casper ES, Brennan MF. Long-term results of a prospective randomized trial of adjuvant brachytherapy in soft tissue sarcoma. J Clin Oncol (1996) 14:859-68.

35. Alektiar KM, Brennan MF, Singer S. Local control comparison of adjuvant brachytherapy to intensity-modulated radiotherapy in primary high-grade sarcoma of the extremity. Cancer (2011) 117:3229-34. doi:10.1002/cncr.25882

Conflict of Interest Statement: The authors declare that the research was conducted in the absence of any commercial or financial relationships that could be construed as a potential conflict of interest.

Received: 05 August 2014; accepted: 28 October 2014; published online: 17 November 2014.

Citation: Kahn J, Gillespie A, Tsokos M, Ondos J, Dombi E, Camphausen K, Widemann BC and Kaushal A (2014) Radiation therapy in management of sporadic and neurofibromatosis type 1-associated malignant peripheral nerve sheath tumors. Front. Oncol. 4:324. doi: 10.3389/fonc.2014.00324

This article was submitted to Radiation Oncology, a section of the journal Frontiers in Oncology.

Copyright $\odot 2014$ Kahn, Gillespie, Tsokos, Ondos, Dombi, Camphausen, Widemann and Kaushal. This is an open-access article distributed under the terms of the Creative Commons Attribution License (CC BY). The use, distribution or reproduction in other forums is permitted, provided the original author $(s)$ or licensor are credited and that the original publication in this journal is cited, in accordance with accepted academic practice. No use, distribution or reproduction is permitted which does not comply with these terms. 